\title{
Effects of Phase II Comprehensive Cardiac Rehabilitation on Risk Factor Modification and Exercise Capacity in Patients With Acute Coronary Syndrome - Results From the JACR Registry -
}

Miho Nishitani-Yokoyama, MD; Hiroyuki Daida, MD; Kazunori Shimada, MD; Akiko Ushijima, MD; Keisuke Kida, MD, PhD; Yuji Kono, PhD;

Yasuhiko Sakata, MD, PhD; Masatoshi Nagayama, MD, PhD; Yutaka Furukawa, MD, PhD; Nagaharu Fukuma, MD, PhD; Keijiro Saku, MD, PhD; Shin-ichiro Miura, MD, PhD;

Yusuke Ohya, MD, PhD; Youichi Goto, MD, PhD; Shigeru Makita, MD, PhD for the Japanese Association of Cardiac Rehabilitation (JACR) Registration Committee

\begin{abstract}
Background: Cardiac rehabilitation (CR) is categorized as a Class I recommendation in guidelines for the management of patients with acute coronary syndrome (ACS); however, nationwide studies on CR in patients with ACS remain limited in Japan.

Methods and Results: The Japanese Association of Cardiac Rehabilitation (JACR) Registry is a nationwide, real-world database for patients participating in CR. From the JACR Registry database, we analyzed 924 patients participating in Phase II CR in 7 hospitals between September 2014 and December 2016. The mean age of patients was $65.9 \pm 12.0$ years, and $80 \%$ were male. The prevalence of ST-elevation myocardial infarction (STEMI), non-STEMI (NSTEMI), and unstable angina pectoris (UAP) was $58 \%$, $9 \%$, and $33 \%$, respectively. The prevalence of hypertension, diabetes, dyslipidemia, current smoking, and a family history was $55 \%$, $27 \%, 67 \%, 21 \%$, and $10 \%$, respectively. Among the entire CR cohort at baseline, $96 \%, 78 \%$, and $92 \%$ were treated with aspirin, $\beta$-blockers, and statins, respectively. After CR, the values of body mass index, the lipid profile, and exercise capacity significantly improved in the STEMI, NSTEMI and UAP groups.
\end{abstract}

Conclusions: In the JACR Registry, a high rate of guideline-recommended medications at baseline and improvements in both coronary risk factors and exercise capacity after $\mathrm{CR}$ were observed in patients with ACS.

Key Words: Acute coronary syndrome; Cardiac rehabilitation; Coronary risk factors; Exercise capacity; Japanese Association of Cardiac Rehabilitation (JACR) Registry

C omprehensive cardiac rehabilitation (CR), including exercise training, has been proven to improve exercise capacity and quality of life and reduce cardiovascular and total mortality in patients with cardiovascular disease (CVD)., ${ }^{1,2}$ The guidelines of the American College of Cardiology, American Heart Association, and Japanese Circulation Society (JCS) recommend the use of CR after acute coronary syndrome (ACS), including ST-elevation myocardial infarction (STEMI), non-STEMI (NSTEMI), and unstable angina pectoris (UAP), as a Class I intervention. ${ }^{\mathbf{3}, 4}$

Received August 7, 2020; revised manuscript received September 29, 2020; accepted October 20, 2020; J-STAGE Advance Publication released online November 27, 2020 Time for primary review: 32 days

Department of Cardiovascular Biology and Medicine, Juntendo University Graduate School of Medicine, Tokyo (M.N.-Y., H.D., K. Shimada); Faculty of Health and Science Juntendo University, Tokyo (H.D.); Department of Cardiovascular Medicine, Tokai University Hachioji Hospital, Tokyo (A.U.); Department of Pharmacology, St. Marianna University School of Medicine, Kawasaki (K.K.); Department of Rehabilitation, Fujita Health University Hospital, Toyoake (Y.K.); Department of Cardiovascular Medicine, Tohoku University School of Medicine, Sendai (Y.S.); Department of Cardiology, Sakakibara Heart Institute, Tokyo (M.N.); Department of Cardiology, Kobe City Medical Center General Hospital, Kobe (Y.F.); School of Nursing, Japanese Red Cross Toyota College of Nursing, Toyota (N.F.); Department of Cardiovascular Medicine, Fukuoka University School of Medicine, Fukuoka (K. Saku, S. Miura); Department of Cardiovascular Medicine, Nephrology and Neurology, Graduate School of Medicine University of the Ryukyus, Okinawa (Y.O.); Yoka Municipal Hospital, Yabu (Y.G.); and Department of Cardiac Rehabilitation, Saitama Medical University International Medical Center, Hidaka (S. Makita), Japan

H.D., Y.S., S. Miura, and Y.O. are members of Circulation Reports' Editorial Team.

Mailing address: Miho Nishitani-Yokoyama, MD, Department of Cardiovascular Biology and Medicine, Juntendo University Graduate School of Medicine, 2-1-1 Hongo, Bunkyo-ku, Tokyo 113-8421, Japan. E-mail: mipocch@juntendo.ac.jp

All rights are reserved to the Japanese Circulation Society. For permissions, please e-mail: cr@j-circ.or.jp

ISSN-2434-0790 


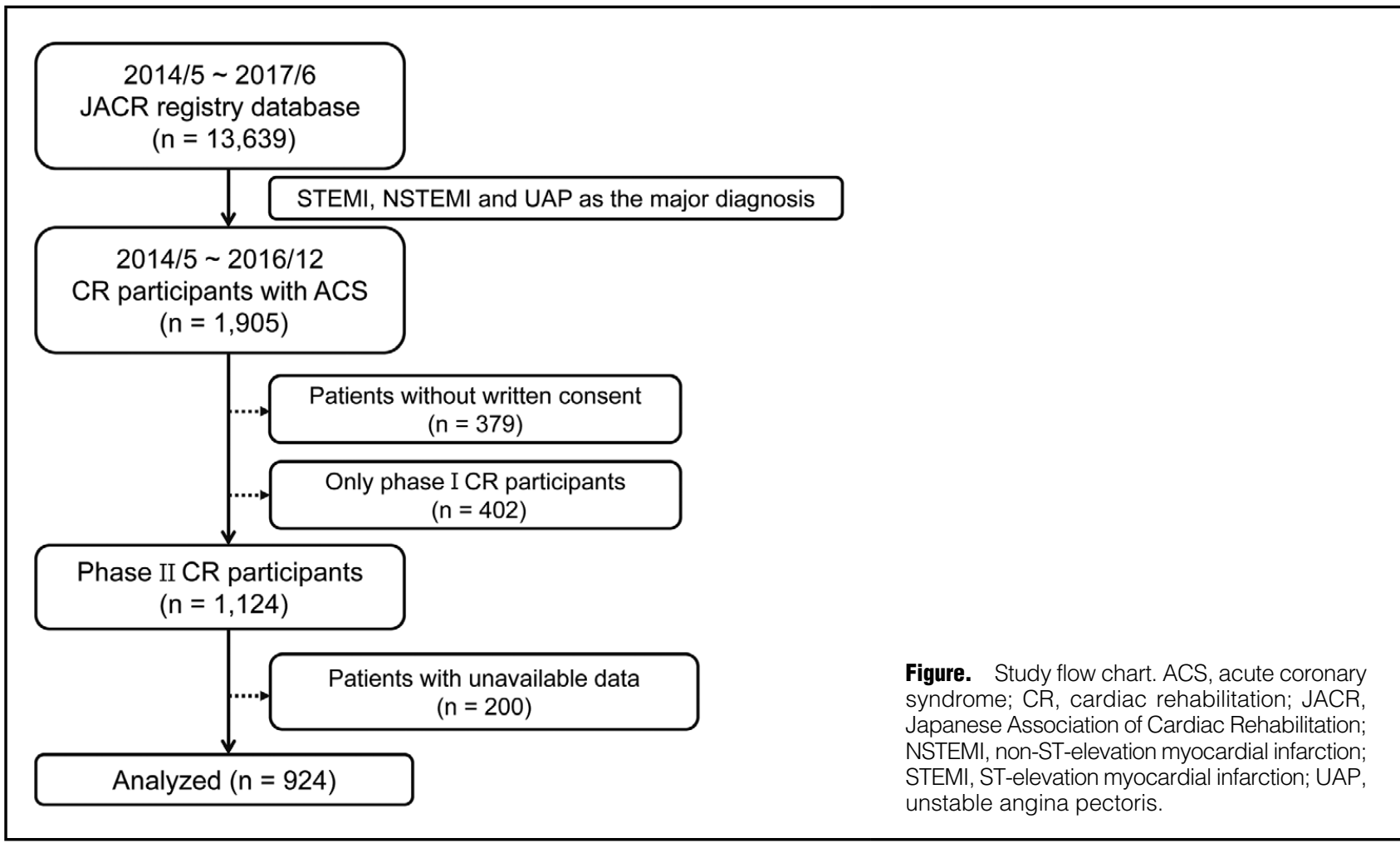

However, in Japan, the implementation of CR remains limited, even among JCS training hospitals. The Japanese Cardiac Rehabilitation Survey found that CR, especially outpatient $\mathrm{CR}$, was insufficient compared with the broad dissemination of percutaneous coronary intervention (PCI). ${ }^{5}$ A recent analysis reported that, in Japan, between $3.8 \%$ and $7.6 \%$ of patients participated in CR. ${ }^{5}$ In May 2014, the Japanese Association of Cardiac Rehabilitation (JACR) set up a JACR Registry, a nationwide and realworld database of patients participating in CR in Japan. The JACR Registry assesses the current status and effects of Phase II CR in patients with CVD using a web-based data collection system. ${ }^{6}$

To date, there have been very few multicenter studies of CR in patients with ACS in Japan. The aim of this study was to assess the effects of Phase II CR in patients with ACS enrolled in the JACR Registry.

\section{Methods}

\section{Study Design and Subjects}

The JACR Registry is a nationwide, real-world, observational database for patients with CVD, including acute myocardial infarction, angina pectoris, heart failure, postcardiac surgery, macrovascular disease, peripheral artery disease, and post-transcatheter aortic valve implantation. All patients in the Registry participated in Phase II CR. The CR time period following hospital admission for ACS was set according to the guidelines as the acute phase, the early recovery phase (early Phase II), late recovery phase (late Phase II), and the maintenance phase. ${ }^{4}$ Early Phase II $\mathrm{CR}$ was defined as a comprehensive program performed in the general cardiovascular ward after the patient had left the coronary care unit to prepare the patient for a return to society. Late Phase II CR was defined as a comprehensive program performed in an outpatient rehabilitation center to prepare the patient for a return the society and help them establish a new lifestyle. ${ }^{4}$

The JACR Registry was designed as a central Internet database connected to every $\mathrm{CR}$ hospital researcher (http://202.212.80.90/regist/login.php). All data were collected electronically using a web-based data entry system. Information was collected on patient characteristics, medical history, comorbidities, coronary risk factors, medications, exercise capacity, mortality, and major adverse cardiac and cerebrovascular events after 1, 3, and 5 years. Sites transmitted data through an electronic case report form (eCRF). The eCRFs were checked by members of the Registry and by the Analysis Committee of the JACR in order to ensure data quality. Informed consent was obtained from all participants according to the mandates of the ethics committee at each participating hospital.

The present study was conducted in accordance with the ethical principles in the Declaration of Helsinki. The study protocol was approved by the ethics committees of the participating institutions. The JACR Registry is registered with the University Hospital Medical Information Network (UMIN) Clinical Trials Registry (ID: UMIN000021647).

In the present study, we assessed the effects of Phase II $\mathrm{CR}$ in patients with ACS enrolled in the JACR Registry. The diagnoses of STEMI, NSTEMI, and UAP were based on the third universal definition of myocardial infarction consensus document, published in $2012 .^{7}$ The participating hospitals meet the standards for CR facilities in Japan and are where a CR instructor works. The CR program, which consisted of exercise training, patient education, and counseling for patients, was based on the guidelines and a standard rehabilitation program from acute to recovery 


\begin{tabular}{|c|c|c|c|c|}
\hline & $\begin{array}{c}\text { All subjects } \\
(n=924)\end{array}$ & $\begin{array}{c}\text { STEMI } \\
(\mathrm{n}=534)\end{array}$ & $\begin{array}{l}\text { NSTEMI-UAP } \\
(\mathrm{n}=390)\end{array}$ & P value ${ }^{A}$ \\
\hline Age (years) & $65.9 \pm 12.0$ & $64.6 \pm 12.6$ & $67.7 \pm 10.9$ & $<0.01$ \\
\hline Male sex & $738(80)$ & $429(80)$ & $309(79)$ & 0.67 \\
\hline Hypertension & $508(55)$ & $264(50)$ & $244(63)$ & $<0.01$ \\
\hline Diabetes & $246(27)$ & $111(21)$ & $135(35)$ & $<0.01$ \\
\hline Dyslipidemia & $620(67)$ & $339(64)$ & $281(72)$ & $<0.01$ \\
\hline Current smoker & $189(21)$ & $130(25)$ & $59(15)$ & $<0.01$ \\
\hline Family history of IHD & $86(10)$ & $50(10)$ & $36(10)$ & 0.98 \\
\hline Ejection fraction (\%) & $54.9 \pm 9.9$ & $51.8 \pm 10.0$ & $57.1 \pm 9.6$ & $<0.01$ \\
\hline \multicolumn{5}{|l|}{ Laboratory data } \\
\hline Hemoglobin (g/dL) & $13.2 \pm 1.8$ & $13.5 \pm 1.8$ & $12.9 \pm 1.7$ & $<0.01$ \\
\hline Creatinine (mg/dL) & $1.0 \pm 1.0$ & $0.96 \pm 0.78$ & $1.08 \pm 1.2$ & 0.55 \\
\hline Total cholesterol (mg/dL) & $171 \pm 44$ & $174 \pm 46$ & $168 \pm 42$ & 0.13 \\
\hline Triglyceride (mg/dL) & $136 \pm 76$ & $136 \pm 75$ & $134 \pm 77$ & 0.65 \\
\hline $\mathrm{HDL}-\mathrm{C}(\mathrm{mg} / \mathrm{dL})$ & $43 \pm 13$ & $42 \pm 12$ & $43 \pm 13$ & 0.38 \\
\hline LDL-C (mg/dL) & $102 \pm 37$ & $103 \pm 38$ & $102 \pm 37$ & 0.97 \\
\hline FBS (mg/dL) & $137 \pm 58$ & $142 \pm 62$ & $127 \pm 50$ & 0.02 \\
\hline $\mathrm{HbA1c}(\%)$ & $6.3 \pm 1.0$ & $6.2 \pm 1.0$ & $6.4 \pm 1.0$ & 0.02 \\
\hline BNP (pg/mL) & 110 [46-239] & 124 [53-245] & 95 [35-201] & 0.06 \\
\hline NT-proBNP (pg/mL) & $494[157-1,084]$ & $588[248-1,345]$ & 364 [130-926] & $<0.01$ \\
\hline CRP (mg/dL) & $1.12 \pm 2.52$ & $1.13 \pm 2.81$ & $1.11 \pm 1.96$ & $<0.01$ \\
\hline hsCRP (mg/dL) & $0.83 \pm 1.57$ & $0.74 \pm 1.42$ & $0.90 \pm 1.67$ & 0.46 \\
\hline \multicolumn{5}{|l|}{ Medications } \\
\hline ASA & $889(96)$ & $518(97)$ & $371(95)$ & 0.14 \\
\hline DAPT & $651(75)$ & $458(86)$ & $233(60)$ & $<0.01$ \\
\hline ACEI & $545(59)$ & $425(80)$ & $120(31)$ & $<0.01$ \\
\hline ARB & $178(19)$ & $61(11)$ & $117(30)$ & $<0.01$ \\
\hline$\beta$-blockers & $720(78)$ & 447 (84) & $273(70)$ & $<0.01$ \\
\hline CCB & $219(24)$ & $80(15)$ & $139(36)$ & $<0.01$ \\
\hline Statin & $851(92)$ & $503(94)$ & $348(89)$ & $<0.01$ \\
\hline OADs & $237(26)$ & $108(20)$ & $129(33)$ & $<0.01$ \\
\hline Insulin & $37(4)$ & $13(2)$ & $24(6)$ & $<0.01$ \\
\hline
\end{tabular}

Data are presented as the mean $\pm \mathrm{SD}$, median [interquartile range], or as $\mathrm{n}(\%)$. AP values are for comparisons of the STEMI vs. NSTEMI-UAP groups. ACEI, angiotensin-converting-enzyme inhibitor; ARB, angiotensin II receptor blocker; ASA, aspirin; BNP, B-type natriuretic peptide; CCB, calcium channel blockers; DAPT, dual antiplatelet therapy; FBS, fasting blood sugar; HDL-C, high-density lipoprotein cholesterol; hsCRP, high-sensitivity C-reactive protein; IHD, ischemic heart disease; LDL-C, low-density lipoprotein cholesterol; NT-proBNP, N-terminal pro B-type natriuretic peptide; NSTEMI-UAP, non-STEMI plus unstable angina pectoris; OADs, oral antidiabetic drugs; STEMI, ST-elevation myocardial infarction.

phase myocardial infarction., ${ }^{4,8}$

The flow diagram of the study is shown in Figure. In all, 13,639 patients were registered and prospectively analyzed between May 2014 and June 2017 from 17 JACR Registry facilities. There were 1,905 patients with ACS from 12 hospitals enrolled during this period. Of those, we excluded 379 patients who did not provide written informed consent, 402 patients who participated only in Phase I CR, and 200 patients with missing clinical data. Finally, 924 patients from 7 hospitals were include in the analysis (Figure).

Further, patients were divided into 2 groups, a STEMI and NSTEMI-UAP (NSTEMI and UAP) group, and the characteristics and baseline and follow-up late Phase $2 \mathrm{CR}$ data were compared between these 2 groups.

\section{eCRF}

The eCRF collected information on patients' baseline characteristics (age, sex, initiating clinical event, history of
CVD risk factors, and medication), actual CR performance, and coronary risk factors (body mass index [BMI], lowdensity lipoprotein cholesterol [LDL-C], high-density lipoprotein cholesterol [HDL-C], triglyceride, fasting glucose, $\mathrm{HbA1c}$ ) as evaluated at $\mathrm{CR}$ baseline and follow-up (after between 3 and 5 months), as well as mortality and major adverse cardiac and cerebrovascular events after 1 , 3 , and 5 years.

\section{Statistical Analysis}

The results are presented as the mean \pm SD and were analyzed using JMP Version 13 for Windows (SAS Institute, Cary, NC, USA). Depending on data distributions, clinical characteristics were compared between groups using unpaired t-tests or Wilcoxon signed-rank tests. Data at baseline and follow-up CR were compared for each patient using paired t-tests to evaluate the singular effects of $C R$. Two-sided $\mathrm{P}<0.05$ was considered significant. 


\begin{tabular}{|c|c|c|c|c|}
\hline \multirow[b]{3}{*}{ Body mass index $\left(\mathrm{kg} / \mathrm{m}^{2}\right)$} & \multicolumn{2}{|c|}{ STEMI $(n=127)$} & \multicolumn{2}{|c|}{ NSTEMI-UAP $(n=94)$} \\
\hline & Baseline & Follow-up & Baseline & Follow-up \\
\hline & $23.7 \pm 3.2$ & $23.3 \pm 2.9^{*}$ & $25.3 \pm 3.2$ & $24.6 \pm 2.9^{\star}$ \\
\hline \multicolumn{5}{|l|}{ Lipid profile and glucose metabolism } \\
\hline LDL-C (mg/dL) & $114 \pm 35$ & $82 \pm 23^{*}$ & $108 \pm 42$ & $87 \pm 23$ \\
\hline HDL-C (mg/dL) & $42 \pm 15$ & $45 \pm 13^{*}$ & $42 \pm 11$ & $47 \pm 12^{*}$ \\
\hline Triglyceride (mg/dL) & $159 \pm 98$ & $125 \pm 47$ & $133 \pm 63$ & $154 \pm 107$ \\
\hline FBS (mg/dL) & $184 \pm 62$ & $102 \pm 11^{*}$ & $122 \pm 40^{\dagger}$ & $161 \pm 86$ \\
\hline $\mathrm{HbA1c}(\%)$ & $6.2 \pm 0.8$ & $6.1 \pm 0.5$ & $6.3 \pm 0.9$ & $6.6 \pm 1.2$ \\
\hline \multicolumn{5}{|l|}{ No. CR programs } \\
\hline Early Phase II CR implementation (n) & & $7.0 \pm 9.7$ & & $11.0 \pm 11.7^{\dagger}$ \\
\hline Late Phase II CR implementation (n) & & $12.8 \pm 12.5$ & & $15.5 \pm 13.2$ \\
\hline Total Phase II CR implementation (n) & & $19.8 \pm 18.7$ & & $26.5 \pm 22.0^{\dagger}$ \\
\hline
\end{tabular}

Data are presented as the mean \pm SD. ${ }^{*} \mathrm{P}<0.05$ compared with baseline; ${ }^{\mathrm{P}} \mathrm{P}<0.05$ compared with the STEMI group. CR, cardiac rehabilitation. Other abbreviations as in Table 1.

\section{Results}

\section{Clinical Characteristics}

Table 1 shows patients' clinical characteristics. The number of patients with a history of STEMI, NSTEMI, and UAP was $534(58 \%), 85(9 \%)$, and $305(33 \%)$, respectively. The mean age of patients was $65.9 \pm 12.0$ years, and $80 \%$ were male. The number of patients with hypertension, diabetes, dyslipidemia, current smoking, and family history of ischemic heart disease was $508(55 \%), 246(27 \%), 620$ $(67 \%), 189(21 \%)$, and $86(10 \%)$, respectively.

Among the entire CR cohort, 889 (96\%), 651 (75\%), 545 $(59 \%), 720(78 \%), 219(24 \%), 851(92 \%), 237(26 \%)$, and 37 $(4 \%)$ patients were treated with aspirin, dual antiplatelet therapy (DAPT), an angiotensin-converting enzyme inhibitor (ACEI), $\beta$-blockers, calcium antagonists, statins, oral diabetes drugs, and insulin, respectively. At baseline, systolic blood pressure, LDL-C, triglycerides, fasting blood glucose, and $\mathrm{HbA} 1 \mathrm{c}$ levels were $122 \pm 22 \mathrm{mmHg}, 102 \pm 37 \mathrm{mg} / \mathrm{dL}$, $136 \pm 76 \mathrm{mg} / \mathrm{dL}, 137 \pm 58 \mathrm{mg} / \mathrm{dL}$, and $6.3 \pm 1.0 \%$, respectively. After Phase 1 and/or early Phase II CR, 434 patients (47\%) participated in late Phase II CR.

\section{Clinical Characteristics in the STEMI vs. NSTEMI-UAP Groups}

Patient characteristics were compared between the STEMI $(\mathrm{n}=534 ; 58 \%)$ and NSTEMI-UAP $(\mathrm{n}=390 ; 42 \%)$ groups (Table 1). Patients in the STEMI group were significantly younger and were more likely to be current smokers than those in the NSTEMI-UAP group $(\mathrm{P}<0.05)$. The prevalence of hypertension, diabetes, and dyslipidemia was significantly higher in the NSTEMI-UAP than STEMI group $(\mathrm{P}<0.05$ for all). B-Type natriuretic peptide (median [interquartile range] $588[248-1,345]$ vs. $364[130,926] \mathrm{pg} / \mathrm{mL})$ and C-reactive protein $(1.13 \pm 2.81$ vs. $1.11 \pm 1.96 \mathrm{mg} / \mathrm{dL})$ levels were higher in the STEMI than NSTEMI-UAP group, whereas ejection fraction was lower in the STEMI than NSTEMI-UAP group $(51.8 \pm 10.0 \%$ vs. $57.1 \pm 9.8 \%$; all $\mathrm{P}<0.05$ ).

The prescription rates of DAPT ( $86 \%$ vs. $60 \%$ ), ACEI $(80 \%$ vs. $31 \%)$, $\beta$-blockers $(84 \%$ vs. $70 \%)$, and statins ( $94 \%$ vs. $89 \%$ ) were significantly higher in the STEMI than NSTEMI-UAP group $(\mathrm{P}<0.05$ for all). Conversely, prescription rates of angiotensin II receptor blockers (30\% vs. $11 \%$ ), calcium channel blockers ( $36 \%$ vs. $15 \%$ ), oral antidiabetes drugs ( $33 \%$ vs. $20 \%)$, and insulin ( $6 \%$ vs. $2 \%)$ were significantly lower in the STEMI than NSTEMI-UAP group $(\mathrm{P}<0.05$ for all).

\section{Coronary Risk Factors at Baseline and After CR in the STEMI vs. NSTEMI-UAP Groups}

We then assessed 221 patients (STEMI, $\mathrm{n}=127$; NSTEMIUAP, n=94) who completed the late Phase II CR and had both initial and final data. Table 2 shows the coronary risk factors at baseline and after CR in both groups. At baseline, BMI did not differ significantly between the 2 groups. During follow-up, BMI decreased significantly in both the STEMI (from $23.7 \pm 3.2$ to $23.3 \pm 2.9 \mathrm{~kg} / \mathrm{m}^{2}$ ) and NSTEMIUAP (from $25.3 \pm 3.2$ to $24.6 \pm 2.9 \mathrm{~kg} / \mathrm{m}^{2}$ ) groups $(\mathrm{P}<0.05$ ). At baseline, there were no significant differences in the lipid profile between the 2 groups. At follow-up, LDL-C level was significantly lower in STEMI group $(\mathrm{P}<0.05)$, whereas HDL-C levels were significantly $(\mathrm{P}<0.05)$ higher, in both groups. In the STEMI group, serum fasting glucose level were significantly lower at follow-up than at baseline $(102 \pm 11$ vs. $184 \pm 62 \mathrm{mg} / \mathrm{dL} ; \mathrm{P}<0.05)$. Significantly more patients in the NSTEMI-UAP than STEMI group took part in Phase II CR (26.5 \pm 22.0 vs. $19.8 \pm 18.7 ; \mathrm{P}=0.02)$.

\section{Exercise Tolerance Parameters at Baseline and Follow-up in the STEMI vs. NSTEMI-UAP Groups}

Table 3 shows the parameters of cardiopulmonary exercise testing (CPET) at baseline and follow-up in the 2 groups. We assessed 254 patients (STEMI, $n=144$; NSTEMI-UAP, $\mathrm{n}=110$ ) who performed both initial and final CPET. At baseline, peak $\dot{\mathrm{VO}}_{2}$ levels were $80 \%$ and $77 \%$ of the age average in the STEMI and NSTEMI-UAP groups, respectively. At follow-up, peak $\dot{\mathrm{V}}_{2}$ levels had increased significantly to almost $90 \%$ of the age average in both groups. Other CPET parameters were also improved in both groups $(\mathrm{P}<0.05)$.

\section{Discussion}

The JACR Registry is a nationwide registry that records the clinical characteristics, treatment, and outcomes of 


\begin{tabular}{|c|c|c|c|c|c|c|}
\hline \multirow[b]{3}{*}{ Baseline } & \multicolumn{3}{|c|}{ STEMI group $(n=144)$} & \multicolumn{3}{|c|}{ NSTEMI-UAP group $(n=110)$} \\
\hline & Baseline & Follow-up & $P$ value & Baseline & Follow-up & $P$ value \\
\hline & & & & & & \\
\hline $\mathrm{SBP}(\mathrm{mmHg})$ & $119 \pm 22$ & $122 \pm 20$ & 0.02 & $127 \pm 20^{*}$ & $127 \pm 21$ & 0.89 \\
\hline HR (beats/min) & $69 \pm 10$ & $66 \pm 9$ & $<0.01$ & $71 \pm 11$ & $67 \pm 10$ & $<0.01$ \\
\hline \multicolumn{7}{|l|}{ AT } \\
\hline $\operatorname{ATV்}_{2}\left(\mathrm{~mL} \cdot \mathrm{min}^{-1} \cdot \mathrm{kg}^{-1}\right)$ & $12.2 \pm 2.3$ & $13.6 \pm 3.1^{\dagger}$ & $<0.01$ & $11.5 \pm 2.6$ & $12.6 \pm 2.4$ & $<0.01$ \\
\hline AT (\% predicted value) & $83 \pm 16$ & $92 \pm 20^{\dagger}$ & $<0.01$ & $78 \pm 19$ & $86 \pm 17$ & $<0.01$ \\
\hline \multicolumn{7}{|l|}{ Peak exercise } \\
\hline $\mathrm{SBP}(\mathrm{mmHg})$ & $169 \pm 27$ & $181 \pm 29$ & $<0.01$ & $174 \pm 207$ & $182 \pm 29$ & $<0.01$ \\
\hline HR (beats/min) & $122 \pm 20$ & $129 \pm 20$ & $<0.01$ & $119 \pm 19$ & $125 \pm 20$ & 0.03 \\
\hline Peak $\dot{V O}_{2}\left(\mathrm{~mL} \cdot \mathrm{min}^{-1} \cdot \mathrm{kg}^{-1}\right)$ & $19.0 \pm 5.0$ & $21.8 \pm 5.7$ & $<0.01$ & $17.6 \pm 4.3$ & $20.4 \pm 4.9$ & $<0.01$ \\
\hline Peak $\mathrm{VO}_{2}(\%$ predicted value $)$ & $80 \pm 18$ & $92 \pm 19$ & $<0.01$ & $77 \pm 19$ & $89 \pm 20$ & $<0.01$ \\
\hline Workload (W) & $97 \pm 37$ & $112 \pm 41$ & $<0.01$ & $89 \pm 30$ & $103 \pm 33$ & $<0.01$ \\
\hline RER & $1.16 \pm 0.11$ & $1.19 \pm 0.14$ & 0.02 & $1.18 \pm 0.13$ & $1.19 \pm 0.10$ & 0.11 \\
\hline$\dot{\mathrm{V} E}$ vs. $\dot{\mathrm{V} C \mathrm{C}_{2}}$ slope & $29.9 \pm 7.1$ & $28.4 \pm 5.8$ & $<0.01$ & $30.3 \pm 5.8$ & $29.1 \pm 4.8$ & 0.04 \\
\hline
\end{tabular}

Data are presented as the mean \pm SD. ${ }^{*} \mathrm{P}<0.05$ compared with the STEMI group at baseline; ${ }^{\dagger} \mathrm{P}<0.05$ compared with the NSTEMI-UAP group at follow-up. AT, anaerobic threshold; $\mathrm{ATVO}_{2}$, anaerobic threshold oxygen consumption; CPET, cardiopulmonary exercise testing; HR, heart rate; peak $\mathrm{VO}_{2}$, peak oxygen consumption (exercise capacity); RER, respiratory exchange ratio; SBP, systolic blood pressure; $\mathrm{VE}$ vs. VंCO slope, slope of the relationship between pulmonary ventilation per minute/carbon dioxide output. Other abbreviations as in Table 1.

patients who participate in Phase II CR in Japan. In this study we investigated the clinical background, coronary risk factors, participation rates for Phase II CR, and exercise capacity in patients with ACS. This is the first multicenter registry study to report the effects of Phase II CR on the modification of coronary risk factors and the improvement in exercise capacity in patients with ACS in Japan.

To date, results from several CR registries have been reported., ${ }^{9,10}$ The European Cardiac Rehabilitation Registry and Database (EuroCaReD) has registered 2,054 patients with coronary artery disease (CAD) from 69 institutions in 12 countries. ${ }^{11}$ The EuroCaReD reports patients' clinical backgrounds, but the programs vary considerably by country and the reported guideline compliance rates were low. ${ }^{11}$ The patients enrolled in the JACR Registry were older and had a higher prevalence of diabetes and a lower prevalence of current smokers compared with patients in the EuroCaReD. ${ }^{11}$ The European Action on Secondary and Primary Prevention by Intervention to Reduce Events (EUROASPIRE) study demonstrated that lifestyle and risk factor management was far from optimal in 8,261 patients with CAD from 131 centers in 27 countries. ${ }^{12}$ Most patients did not achieve their blood pressure, lipid, and glycemic targets in line with ESC 2016 guidelines, although the use of cardioprotective medications was high. ${ }^{\mathbf{1 2}}$ In Japan, metabolic risk factors have become more prevalent due to Westernization of the lifestyle and urbanization. This raises a concern that the incidence of CAD in Japan will increase over time. Indeed, recent epidemiological studies suggest that the incidence of acute myocardial infarction (AMI) is increasing in Japan. ${ }^{13}$

The Japan Atherosclerosis Society Guidelines for Prevention of Atherosclerotic Disease (2017) recommend an LDL-C level $<100 \mathrm{mg} / \mathrm{dL}$ for secondary prevention in patients with CAD. ${ }^{14}$ The LDL-C target for high-risk groups, such as those with familial hypercholesterolemia, ACS, or diabetes, is $<70 \mathrm{mg} / \mathrm{dL} .{ }^{14}$ However, in Japan, the "real-world" achievement rate for the optimal LDL-C level for secondary prevention in patients with CAD is deemed to be low. ${ }^{15}$ In the present study, the target LDL-C level was almost achieved at follow-up. Among all participants in late Phase II CR, 89\% used strong statins and 3\% used other lipid-lowering drugs. The same percentage of participants in late Phase II CR used statins during the follow-up. Although the statin dose was unknown, lipid profiles may be improved by a comprehensive prevention program consisting of diet, physical activity, and optimal medical therapy based on the guidelines. Patients in the NSTEMI-UAP group were significantly older and had a higher prevalence of hypertension, diabetes, and dyslipidemia than those in the STEMI group, and a higher number of individuals in the NSTEMI-UAP group were undergoing early Phase II CR implementation (all $\mathrm{P}<0.05$ ). The Osaka Acute Coronary Insufficiency Study reported that independent predictors of longer hospital stay were increased age, diabetes, congestive heart failure, and stroke. ${ }^{16}$ Although the length of hospital stay was unknown in the present study, clinical factors, such as age and the prevalence of coronary risk factors, may explain the larger number patients undergoing early Phase II CR implementation in the NSTEMI-UAP than STEMI group. In addition, there were no significant changes in $\mathrm{HbAlc}$ between baseline and the follow-up examinations in the 2 groups. However, in the STEMI group, fasting blood sugar levels at baseline were higher than at follow-up. Previous studies demonstrated that acute hyperglycemia was a common feature during the early phase after acute ACS, regardless of a patient's diabetes status. ${ }^{17}$ Various mechanisms, such as sympathetic nerve hyperactivity, may cause hyperglycemia.

The Arbeitsgemeinschaft für ambulante kardiologische Rehabilitation (AGAKAR) study analyzed 2,822 patients with various CVD who underwent Phase II or Phase III $\mathrm{CR}$ as outpatients in 8 facilities in Austria. ${ }^{\mathbf{1 8}}$ The AGAKAR registry demonstrated that short- and long-term outpatient $\mathrm{CR}$ is beneficial for coronary risk control, exercise capacity, and quality of life. Exercise therapy for patients with CVD increases their exercise capacity by $15-25 \%$, regardless of cardiac function. ${ }^{\mathbf{2}, \mathbf{4}}$ In the present study, we obtained 
detailed data of exercise capacity following CPET in both the STEMI and NSTEMI-UAP groups, finding a significant improvement in exercise capacity (STEMI group, 15\%; NSTEMI-UAP group, 16\%), which is an important prognostic factor in patients with CVD. ${ }^{19}$

Recently, the widespread use of primary PCI has enabled early ambulation of patients with ACS, reducing acute-phase complications and resulting in minimal physical deconditioning. This shortening of hospitalization has made it difficult to do sufficient early Phase II CR programs with exercise training and patient education. As a result, many patients with AMI are discharged from the hospital early without participating in a CR program. ${ }^{5}$ Kamakura et al reported that patients at low prognostic risk for ACS were younger, exhibited successful reperfusion, and had no history of congestive heart failure. ${ }^{20}$ Individuals with a preserved ejection fraction had a higher prevalence of multiple coronary risk factors than non-low-risk patients. Active participation in late Phase II CR was associated with improved exercise capacity and better coronary risk factor profiles. ${ }^{20}$ The impact of adherence to CR on outcome was clear in patients with CAD, including ACS, who were treated using the guideline-recommended optimal medical therapy. ${ }^{21}$ Previous studies demonstrated that there seemed to be a "dose response curve" for various CR effects with full completion of CR..$^{22}$ The subjects of the present study participated in late Phase II CR, which included supervised exercise therapy at least once a week. Exercise intensity was prescribed individually at a specific anaerobic threshold level as measured by an ergometer test using expiratory gas analysis or a rating of 11-13 on the standard Borg's perceived exertion scale. The program was conducted by CR instructors who followed the guidelines. ${ }^{4,8}$ The menu contents appeared to be sufficient for improving exercise capacity and coronary risk factors during follow-up examinations. Lloyd-Jones et al demonstrated that young patients with any coronary risk factors, despite the low short-term risk, had a higher "lifetime risk for CAD" and greater progression of subclinical coronary atherosclerosis compared with those at low lifetime risk. ${ }^{23}$ Optimal medical therapy and the management of coronary risk factors are necessary for secondary prevention of major adverse cardiac and cerebrovascular events in patients with ACS.24,25 These previous studies suggested that CR was useful and necessary for patients with ACS, although PCI has become widespread.

The clinical registry provides an important tool for monitoring CR program quality. The JACR Registry is establishing the feasibility of a web-based eCRF to collect clinical baseline and outcome parameters in patients who participate in Phase II CR and to assess guideline adherence and CR quality to improve prognosis in patients with CVD in Japan. As the next step, it will be important to analyze the prognosis and investigate the long-term effects of Phase II CR.

\section{Study Limitations}

This study has several limitations. First, the JACR Registry was set up as a nationwide survey; however, data from only 7 hospitals, which were the core facilities of Japanese $\mathrm{CR}$, were analyzed in the present study. Therefore, these data may not be a representative nationwide record. Second, the data were collected from patients who completed the late Phase II CR program. However, we believed that Phase II CR is an effective and important therapeutic strategy for managing coronary risk factors and improving exercise capacity, whether or not the patient experienced a STEMI or NSTEMI event.

\section{Conclusions}

The JACR Registry is the first nationwide registry that includes patient characteristics, CR indications, treatment, and outcomes associated with patients who participate in Phase II CR in Japan. In the JACR Registry, a high rate of guideline-recommended medications at baseline and improvements in both coronary risk factors and exercise capacity after CR were observed in patients with ACS.

\section{Acknowledgments}

The authors thank Mayuko Ichikawa, Takeshi Nagasono, Yuji Nishizaki, Muneko Nojiri, Kyoko Fukataki, Chikako Hayashi, Eriko Ueda, and Sawako Oosawa for their assistance with this work. The authors acknowledge the contributions made by participants of the JACR Registry in the 17 JACR Registry facilities to managing the registry. Participants of the JACR Registry in the 17 JACR Registry facilities are listed in the Appendix.

\section{Sources of Funding}

This registry system and work were supported by the JACR.

\section{Disclosures}

H.D., Y.S., S. Miura, and Y.O. are members of Circulation Reports' Editorial Team. The remaining authors have no conflicts of interest to declare.

\section{IRB Information}

The study protocol was approved by Juntendo Hospital Ethics Committee (No. 13-058) and the ethics committees of participating institutions.

\section{Data Availability}

The datasets will not be publicly available because patient consent in each institute does not allow for such publication. The corresponding author will respond to inquiries regarding data analyses.

\section{References}

1. Braverman DL. Cardiac rehabilitation: A contemporary review. Am J Phys Med Rehabil 2011; 90: 599-611.

2. O'Connor GT, Buring JE, Yusuf S, Goldhaber SZ, Olmstead EM, Paffenbarger RS Jr, et al. An overview of randomized trials of rehabilitation with exercise after myocardial infarction. Circulation 1989; 80: 234-244.

3. Smith SC, Allen J, Blair SN, Bonow RO, Brass LM, Fonarow GC, et al. AHA/ACC guidelines for secondary prevention for patients with coronary and other atherosclerotic vascular disease: 2006 update. Circulation 2006; 113: 2363-2372.

4. JCS Joint Working Group. Guidelines for rehabilitation in patients with cardiovascular disease (JCS 2012): Digest version. Circ J 2014; 78: 2022-2093.

5. Goto Y, Saito M, Iwasaka T, Daida H, Kohzuki M, Ueshima K, et al. Poor implementation of cardiac rehabilitation despite broad dissemination of coronary interventions for acute myocardial infarction in Japan: A nationwide survey. Circ J 2007; 71: $173-179$.

6. Shimada K, Daida H, Yokoyama M, Ushijima A, Kida K, Kono Y, et al. The Japanese Association of Cardiac Rehabilitation (JACR) Registry: The establishment and a first interim analysis (in Japanese). Tokyo, JACR (in press).

7. Thygesen K, Alpert JS, Jaffe AS, Simoons ML, Chaitman BR, White HD, et al. Third universal definition of myocardial infarction. Am J Coll Cardiol 2012; 60: 1581-1598.

8. Japanese Association of Cardiac Rehabilitation Standard Cardiac Rehabilitation Program Writing Committee. Cardiac Rehabilitation Standard Program for Acute Myocardial Infarction (2013) from the Japanese Association of Cardiac Rehabilitation 
- in the recovery phase of myocardial infarction. http://www.jacr. $\mathrm{jp} / \mathrm{web} / \mathrm{wp}$-content/uploads/2015/08/Standard-Program1.pdf (accessed July 26, 2020).

9. Poffley A, Thomas E, Grace SL, Neubeck L, Gallagher R, Niebauer J, et al. A systematic review of cardiac rehabilitation registries. Eur J Prev Cardiol 2017; 24: 1596-1609.

10. Davos $\mathrm{CH}$. Seeking best practices for cardiac rehabilitation registries in Europe. Eur J Prev Cardiol 2017; 24: 1925-1926.

11. Benzer W, Rauch B, Schmid JP, Zwisler AD, Dendale P, Davos $\mathrm{CH}$, et al. Exercise-based cardiac rehabilitation in twelve European countries results of the European cardiac rehabilitation registry. Int J Cardiol 2017; 228: 58-67.

12. Kotseva K, De Backer G, De Bacquer D, Rydén L, Hoes A, Grobbee D, et al. Lifestyle and impact on cardiovascular risk factor control in coronary patients across 27 countries: Results from the European Society of Cardiology ESC-EORP EUROASPIRE V registry. Eur J Prev Cardiol 2019; 26: 824-835.

13. Cui Y, Hao K, Takahashi J, Miyata S, Shindo T, Nishimiya K, et al. Age-specific trends in the incidence and in-hospital mortality of acute myocardial infarction over 30 years in Japan: Report from the Miyagi AMI Registry Study. Circ J 2017; 81: 520 528.

14. Kinoshita M, Yokote $\mathrm{K}$, Arai H, Iida M, Ishigaki Y, Ishibashi S, et al. Japan Atherosclerosis Society (JAS) guidelines for prevention of atherosclerotic cardiovascular diseases 2017. J Atheroscler Thromb 2018; 25: 846-984.

15. Teramoto T, Uno K, Miyoshi I, Khan I, Gorcyca K, Sanchez RJ, et al. Low-density lipoprotein cholesterol levels and lipid-modifying therapy prescription patterns in the real world: An analysis of more than 33,000 high cardiovascular risk patients in Japan. Atherosclerosis 2016; 251: 248-254.

16. Kinjo K, Sato H, Nakatani D, Mizuno H, Shimizu M, Hishida E, et al. Predictors of length of hospital stay after acute myocardial infarction in Japan. Circ J 2004; 68: 809-815.

17. Ishihara M. Acute hyperglycemia in patients with acute myocardial infarction. Circ J 2012; 76: 563-571.

18. Niebauer J, Mayr K, Harpf H, Hofmann P, Müller E, Wonisch $\mathrm{M}$, et al. Long-term effects of outpatient cardiac rehabilitation in Austria: A nationwide registry. Wien Klin Wochenschr 2014; 126: $148-155$.

19. Myers J, Prakash M, Froelicher V, Do D, Partington S, Atwood JE. Exercise capacity and mortality among men referred for exercise testing. $N$ Engl J Med 2002; 346: 793-801.

20. Kamakura T, Kawakami R, Nakanishi M, Ibuki M, Ohara T, Yanase M, et al. Efficacy of out-patient cardiac rehabilitation in low prognostic risk patients after acute myocardial infarction in primary intervention era. Circ J 2011; 75: 315-321.

21. Sunamura M, Ter Hoeve N, van den Berg-Emons RJG, Boersma
E, van Domburg RT, Geleijnse ML. Cardiac rehabilitation in patients with acute coronary syndrome with primary percutaneous coronary intervention is associated with improved 10-year survival. Eur Heart J Qual Care Clin Outcomes 2018; 4: 168-172.

22. Pardaens S, Willems AM, Clays E, Baert A, Vanderheyden M, Verstreken S, et al. The impact of drop-out in cardiac rehabilitation on outcome among coronary artery disease patients. Eur J Prev Cardiol 2017; 14: 1490-1497.

23. Lloyd-Jones DM, Leip EP, Larson MG, D'Agostino RB, Beiser A, Wilson PW, et al. Prediction of lifetime risk for cardiovascular disease by risk factor burden at 50 years of age. Circulation 2006; 113: $791-798$.

24. Mukherjee D, Fang J, Chetcuti S, Moscucci M, Kline-Rogers E, Eagle KA. Impact of combination evidence-based medical therapy on mortality in patients with acute coronary syndromes. Circulation 2004; 109: 745-749.

25. Milani RV, Lavie CJ. Prevalence and profile of metabolic syndrome in patients following acute coronary events and effects of therapeutic lifestyle change with cardiac rehabilitation. Am J Cardiol 2003; 92: 50-54.

\section{Appendix}

Participants of the JACR Registry in the 17 JACR Registry facilities are listed below.

Kentaro Hori and Reina Uewaki (Sakakibara Heart Institute, Fuchu); Michio Nakanishi and Harumi Konishi (National Cerebral and Cardiovascular Center, Suita); Hitoshi Adachi and Yoshihisa Namasu (Gunma Prefectural Cardiovascular Center, Maebashi); Tomoaki Hama (Tokai University Hachioji Hospital, Hachioji); Masahiro Kohzuki and Takahiro Miura (Tohoku Universiry Graduate School of Medicine, Sendai); Hideki Origuchi and Hiroko Kabashima (Japan Community Health care Organizaion Kyushu Hospital, Kitakyushu); Shinichi Momomura and Keisuke Ugata (Jichi Medical University Saitama Medical Center, Saitama); Hideo Izawa (Fujita Health University, Nagoya); Hisashi Kai and Atsushi Katoh (Kurume University Medical Center, Kurume); Hiroshi Yokoya (Asahi General Hospital, Asahi); Junya Ako and Emi Maekawa (Kitasato University School of Medicine), Sagamihara; Minako Yamaoka-Tojo (Kitasato University School of Allied Health Sciences, Sagamihara); Takashi Yokota (Clinical Research and Medical Innovation Center, Hokkaido University Hospital, Sapporo); Arata Fukushima (Hokkaido University, Sapporo); Koichi Tamita, Kazuya Miyamoto, and Nobushige Akagi (Nishinomiya Watanabe Cardiovascular Center, Nishinomiya); Takeshi Kitai and Kentaro Iwata (Kobe City Medical Center General Hospital, Kobe); Hitoshi Anzai (SUBARU Health Insurance Ota Memorial Hospital, Ota); Shinya Minatoguchi and Kazuhiko Nishigaki (Gifu University, Gifu) 